\title{
EXCEPTIONAL SETS FOR INNER FUNCTIONS
}

\section{RENATE McLAUGHLIN}

\section{Introduction}

A function $f(z)$, defined and analytic in the unit disk $D=\{z:|z|<1\}$, is an inner function if $|f(z)| \leqslant 1(z \in D)$ and if $\lim _{r \rightarrow 1}\left|f\left(r e^{i \theta}\right)\right|=1$ for almost all $\theta(0 \leqslant \theta<2 \pi)$. Every inner function $f$ can be factored into a Blaschke product and a singular inner function, where the Blaschke product

$$
B(z)=\prod_{k=1}^{\infty} \frac{\bar{a}_{k}}{\left|a_{k}\right|} \frac{a_{k}-z}{1-\bar{a}_{k} z}
$$

is constructed from the zeros of $f$ and the singular inner factor

$$
S(z)=e^{i \theta} \exp \left\{-\int_{0}^{2 \pi} \frac{e^{i t}+z}{e^{i t}-z} d \mu(t)\right\}
$$

has no zeros in $D$ (the positive measure $\mu$ is supported on a set of Lebesgue measure zero). To avoid trivial cases, we assume throughout this note that $f$ is neither a constant nor a finite Blaschke product.

Two exceptional sets are associated with each inner function $f$ :

$$
E_{1}(f)=\{w:|w|<1, f \text { assumes } w \text { at most finitely often }\}
$$

and

$$
E_{2}(f)=\left\{w:|w|<1, \frac{f(z)-w}{1-\bar{w} f(z)} \text { is not a Blaschke product }\right\}
$$

Clearly, the inclusion $E_{1}(f) \subseteq E_{2}(f)$ holds for each inner function $f$. It is known that the sets $E_{1}$ and $E_{2}$ have capacity zero. Moreover, if $E$ is a closed subset of the open unit disk of capacity zero, then there exists an inner function $f$ such that $E=E_{1}(f)[1 ;$ p. 37]. We show that this function $f$ can be chosen in such a way that $E=E_{1}(f)=E_{2}(f)$.

We call

$$
M_{a}(f(z))=\frac{f(z)-a}{1-\bar{a} f(z)} \quad(|a|<1)
$$

a Möbius transform of $f$. Since, for each inner function $f$, the exceptional set $E_{2}(f)$ has capacity zero, each inner function is a Möbius transform of a Blaschke product. It is natural to ask for Blaschke products all of whose Möbius transforms are again Blaschke products. We call these Blaschke products indestructible.

In this note, we characterize indestructible Blaschke products, and we derive a criterion that allows us to construct indestructible Blaschke products. In the last

Received 6 July, 1970.

This research was supported in part by the National Science Foundation.

[J. London Math. Soc. (2), 4 (1972), 696-700] 
section, we study some relationships between the sets $E_{1}$ and $E_{2}$ by dividing the collection of inner functions into equivalence classes.

I am indebted to George Piranian and Allen L. Shields for several helpful conversations.

\section{Indestructible Blaschke products}

Definition. An inner function $f$ is an indestructible Blaschke product if $E_{2}(f)=\varnothing$.

An indestructible Blaschke product is also a Blaschke product, because $0 \notin E_{2}$. Since $E_{1} \subseteq E_{2}$, an indestructible Blaschke product assumes each point of the unit disk infinitely often. The converse fails: if a Blaschke product assumes each point of the unit disk infinitely often, then it need not be indestructible.

LEMMA 1. Choose an inner function $f$ and a point $a(|a|<1)$ such that $f(0) \neq a$. Suppose $a_{1}, a_{2}, \ldots\left(0<\left|a_{1}\right| \leqslant\left|a_{2}\right| \leqslant \ldots\right)$ are the solutions of the equation $f(z)=a$. Then

$$
\left|\frac{f(0)-a}{1-\bar{a} f(0)}\right| \leqslant \prod_{j}\left|a_{j}\right|
$$

and equality holds if and only if $M_{a}(f(z))$ is a Blaschke product.

Proof. Since $M_{a}(f(z))$ is an inner function, we can write $M_{a}(f(z))=B(z) S(z)$, where $B(z)$ is constructed from the zeros of $f(z)-a$. Obviously, $|S(0)| \leqslant 1$. Hence

$$
\left|M_{a}(f(0))\right|=|B(0) S(0)| \leqslant|B(0)|=\prod_{j}\left|a_{j}\right| .
$$

Clearly, equality holds if and only if $|S(0)|=1$, and $|S(0)|=1$ if and only if $S(z)$ is a constant of modulus 1 .

Suppose $f$ is an inner function with $f(0)=0$, and let $a_{1}, a_{2}, \ldots$ denote the $a$-points $(a \neq 0)$ of $f$. Lemma 1, together with the fact that the set $E_{2}$ has capacity zero, implies that

$$
|a|=\prod_{j}\left|a_{j}\right|
$$

for almost all $a(0<|a|<1)$.

Suppose $B(z)$ is a Blaschke product. Put $B(0)=b$, and suppose

$$
B(z)-b=b_{n} z^{n}+b_{n+1} z^{n+1}+\ldots\left(b_{n} \neq 0\right) .
$$

For $a \neq b$, let $a_{1}, a_{2}, \ldots$ denote the $a$-points of $B$, and let $z_{1}, z_{2}, \ldots$ denote the non-zero solutions of the equation $B(z)-b=0$.

THEOREM 1. The Blaschke product $B(z)$ is indestructible if and only if

$$
\left|\frac{b-a}{1-\bar{a} b}\right|=\prod_{j=1}^{\infty}\left|a_{j}\right| \quad(|a|<1, \quad a \neq b)
$$

and

$$
\frac{\left|b_{n}\right|}{1-|b|^{2}}=\prod_{j=1}^{\infty}\left|z_{j}\right|
$$

The proof is obvious. 
While it is nice to have a necessary and sufficient condition, the condition in Theorem 1 does not tell us how to construct an indestructible Blaschke product. In order to obtain a "practical" (but only sufficient) condition, we recall some known results.

Proposition 1 (W. Seidel [4; Theorem 11, p. 215]). Suppose $f(z)$ is an inner function. If $f$ assumes the value $a(|a|<1)$ infinitely often and if the relation

$$
\lim _{r \rightarrow 1} f\left(r e^{i \theta_{0}}\right)=a
$$

holds for no radius $\theta=\theta_{0}$, then $M_{a}(f(z))$ is a Blaschke product; in other words,

$$
\frac{f(z)-a}{1-\bar{a} f(z)}=e^{i \psi} B(z)
$$

If $e^{i \theta}$ is not a limit point of zeros of the Blaschke product $B(z)$, then clearly

$$
\lim _{r \rightarrow 1}\left|B\left(r e^{i \theta}\right)\right|=1 \text {. }
$$

We can now prove the following practical condition, which allows us to construct indestructible Blaschke products.

THEOREM 2. Suppose $B(z)$ is a Blaschke product. If there exists no radius along which $B(z)$ tends to a limit a with $0<|a|<1$, then $B(z)$ is indestructible.

Proof. Suppose $B(z)$ assumes a value $w(|w|<1)$ at most finitely often. Then there exists $t(0 \leqslant t<2 \pi)$ such that $\lim _{r \rightarrow 1} B\left(r e^{i t}\right)=w[3 ;$ p. 37], a contradiction. Hence $B(z)$ assumes each $w(|w|<1)$ infinitely often, and the theorem now follows from Proposition 1.

(Note that there exist Blaschke products whose radial limits exist and have modulus 1 everywhere.)

CoRollary. The set of indestructible Blaschke products for which all radial limits exist and have modulus 1 or 0 is closed under multiplication.

The condition of Theorem 2 is not necessary. For let $B_{0}(z)$ denote a Blaschke product that admits 0 as radial limit and for which all radial limits exist and have modulus 0 or 1 . By Theorem $2, B_{0}(z)$ is indestructible. Choose $b(0<|b|<1)$. Then $M_{b}\left(B_{0}(z)\right)=B_{1}(z)$ is a Blaschke product. Moreover, since

$$
M_{a}\left(B_{1}(z)\right)=M_{a}\left(M_{b}\left(B_{0}(z)\right)\right)=\frac{1+a \bar{b}}{1+\bar{a} b} M_{s}\left(B_{0}(z)\right),
$$

where $s=(a+b) /(1+a \bar{b}), B_{1}(z)$ is also indestructible. But $B_{1}(z)$ admits $-b$ as radial limit.

\section{Equivalence Classes}

In the previous section, we studied inner functions with the property that

$$
E_{1}=E_{2}=\varnothing \text {. }
$$


In order to study inner functions whose exceptional sets are not necessarily empty, it is convenient to divide the collection of inner functions into equivalence classes.

For each inner function $g$, consider the class of functions

$$
C_{g}=\left\{e^{i \theta} M_{a}(g(z)):|a|<1, \quad 0 \leqslant \theta<2 \pi\right\} .
$$

Define $f \sim g$ if and only if $f \in C_{g}$. It is easy to show that $\sim$ defines an equivalence relation.

Note that each equivalence class contains infinitely many distinct functions, because $e^{i \theta} M_{a}(h(z)) \neq h(z)$ unless $h$ is constant or $a=\theta=0$.

The following result is obvious.

Lemma 2. If an equivalence class $C_{g}$ contains an indestructible Blaschke product, then every element of $C_{g}$ is an indestructible Blaschke product.

Suppose $g(z)$ assumes each value $w(|w|<1)$ infinitely often. Then, clearly, each function $f \in C_{g}$ assumes each value infinitely often. In other words, if $E_{1}(g)=\varnothing$, then $E_{1}\left(e^{i \theta} M_{a}(g)\right)=\varnothing$ for each $\theta$ and each $a(0 \leqslant \theta<2 \pi,|a|<1)$.

LEMmA 3. (i) If $E_{1}(g) \neq \varnothing$ and if $f=e^{i \theta} M_{a}(g)$, then

$$
E_{1}(f)=\left\{e^{i \theta} \frac{w-a}{1-\bar{a} w}: w \in E_{1}(g)\right\} .
$$

(ii) If $E_{2}(g) \neq \varnothing$ and if $f=e^{i \theta} M_{a}(g)$, then

$$
E_{2}(f)=\left\{e^{i \theta} \frac{w-a}{1-\bar{a} w}: w \in E_{2}(g)\right\} .
$$

Proof.

$$
\frac{f(z)-e^{i \theta} M_{a}(w)}{1-e^{-i \theta} \overline{M_{a}(w)} f(z)}=e^{i \theta} \frac{1-a \bar{w}}{1-\bar{a} w} \frac{g(z)-w}{1-\bar{w} g(z)} .
$$

The following result shows that a Blaschke product with distinct zeros and a Blaschke product with repeated zeros can belong to the same equivalence class.

THEOREM 3. Suppose $B_{1}(0) \neq 0$. The Blaschke products $B_{1}$ and $B_{2}$ are equivalent if and only if there exists a number $c(|c|<1)$ such that the zeros of $B_{1}$ coincide with the c-points of $B_{2}$ and $\left|B_{1}(0)\right|=\left|B_{2}(0)-c\right| /\left|1-\bar{c} B_{2}(0)\right|$.

Proof. Suppose $B_{1}(z)=\Pi\left[\left(a_{k}-z\right) /\left(1-\bar{a}_{k} z\right)\right]\left(\bar{a}_{k}|| a_{k} \mid\right)$.

If $B_{1}$ and $B_{2}$ are equivalent, there exist numbers $c(|c|<1)$ and $\theta(0 \leqslant \theta<2 \pi)$ such that $B_{1}(z)=e^{i \theta} M_{c}\left(B_{2}(z)\right)$.

Conversely, suppose there exists a number $c(|c|<1)$ such that $a_{1}, a_{2}, \ldots$ are the zeros of $B_{1}$ and the $c$-points of $B_{2}$ (with the same multiplicities). Then

$$
M_{c}\left(B_{2}(z)\right)=B_{1}(z) S(z) \text {, and }\left|B_{2}(0)-c\right| /\left|1-\bar{c} B_{2}(0)\right|=\left|B_{1}(0) S(0)\right| \text {. }
$$

It follows that $|S(0)|=1$ (recall that $B_{1}(0) \neq 0$ ); hence $S(z) \equiv e^{i \theta}$.

The following lemma shows that, in general, the inclusion $E_{1} \subset E_{2}$ is strict.

LEMMA 4. If the inner function $f$ belongs to an equivalence class containing a function $B(z) S(z)$, where the singular factor $S$ is non-constant and the Blaschke factor $B$ has infinitely many zeros, then the inclusion $E_{1}(f) \subset E_{2}(f)$ is strict. 
(Note that strict inclusion $E_{1}(g) \subset E_{2}(g)$ also holds for each $g \in C_{f}$.)

Proof. There exists an $a(|a|<1)$ such that $e^{i \theta} M_{a}(f(z))=B(z) S(z)$. Hence $a \in E_{2}(f)$, but $a \notin E_{1}(f)$.

The following remarks can be proved easily.

Remarks. 1. An inner function $f$ with $E_{1}(f)=E_{2}(f)$ belongs to an equivalence class containing only Blaschke products, singular inner functions, and products of singular functions with finite Blaschke products.

2. An inner function $f$ for which $E_{1}(f)=E_{2}(f)$ and $E_{1}(f)$ consists of omitted values only, belongs to an equivalence class containing only Blaschke products and purely singular functions.

3. An inner function $f$ with $E_{1}(f)=\varnothing$ and $E_{2}(f) \neq \varnothing$ belongs to an equivalence class each of whose functions has a Blaschke factor with infinitely many zeros. (In other words, a Blaschke product that assumes every value infinitely often need not be indestructible, but the (infinite) Blaschke factor cannot be removed by a Möbius transform.)

4. An equivalence class contains a purely singular inner function if and only if each function in the class omits a value.

The last remark implies that the function $z \exp ((z+1) /(z-1))$ is not equivalent to a purely singular inner function.

We conclude with a theorem that sheds some light on the types of sets that can serve as exceptional sets $E_{2}$.

THEOREM 4. Suppose E is some closed subset of capacity zero in the open unit disk. Then there exists an inner function $f$ for which

$$
E=E_{1}(f)=E_{2}(f) .
$$

Proof. There exists an inner function $f$, mapping the unit disk onto the universal covering surface of the domain $\{|w|<1\}-E$, with the properties that

(i) $E_{1}(f)=E$ (in fact, $f$ omits every value in $E$ ), and

(ii) the set $E$ consists of all radial limits of $f$ whose absolute value is less than 1 (see [2]). We claim that this function $f$ also has the property that $E_{2}(f)=E$. Clearly, $E \subseteq E_{2}(f)$. Now let $a \in E_{2}(f)$, so that the function $g(z)=M_{a}(f(z))$ is not a Blaschke product. Then $g(z)$ admits 0 as radial limit [3; p. 33]; but this means $f(z)$ admits $a$ as radial limit. Hence $a \in E$, by property (ii).

\section{References}

1. E. F. Collingwood and A. J. Lohwater, The Theory of cluster sets (Cambridge University Press, Cambridge, 1966).

2. G. Hössjer and O. Frostman, "Über die Ausnahmestellen eines Blaschkeproduktes ", K. Fysiogr. Sällsk. Lund Förh., 3 (1933), no. 16, 8 pp.

3. K. Noshiro, Cluster sets (Springer-Verlag, Berlin-Göttingen-Heidelberg, 1960).

4. W. Seidel, "On the distribution of values of bounded analytic functions", Trans. Amer. Math. Soc., 36 (1934), 201-226.

The University of Michigan,

Flint College. 